\title{
ON THE EQUICONVERGENCE OF FOURIER SERIES AND FOURIER INTEGRALS
}

\section{FÉLIX EDUARDO HERRERA}

Given any function $f(x)$ defined for $-\infty<x<\infty$, let

$$
\int_{0}^{\infty}\{a(u) \cos u x+b(u) \sin u x\} d u
$$

be the Fourier integral (F.i.) of $f(x)$. Here

$$
a(u)=\frac{1}{\pi} \int_{-\infty}^{\infty} f(t) \cos u t d t ; \quad b(u)=\frac{1}{\pi} \int_{-\infty}^{\infty} f(t) \sin u t d t
$$

are the cosine and sine transforms of $f(x)$. They exist as absolutely convergent integrals if $f \in L(-\infty, \infty)$, or in a certain generalized sense, if $f \in L^{p}(-\infty, \infty)$ with $1<p \leqq 2$.

In all these cases, the partial integrals of (1) are given by the formula

$$
\begin{aligned}
S_{\omega}(x, f) & =\int_{0}^{\omega}\{a(u) \cos u x+b(u) \sin u x\} d u \\
& =\frac{1}{\pi} \int_{-\infty}^{\infty} f(x+t) \frac{\sin \omega t}{t} d t,
\end{aligned}
$$

but the last integral has meaning if

$$
\int_{-\infty}^{\infty} \frac{|f(t)|}{1+|t|} d t<\infty
$$

even if the transforms (2) do not exist.

Now, it is well known that the problem of the representation of $f(x)$ as the limit of

$$
S_{\omega}(x, f)=\frac{1}{\pi} \int_{-\infty}^{\infty} f(x+t) \frac{\sin \omega t}{t} d t
$$

for $\omega \rightarrow \infty$ can be reduced to the representation of $f(x)$ by a Fourier series (F.s.). More precisely, we have the following theorem:

(A) Given any function satisfying (3), and any interval $I_{a}=(a, a+2 \pi)$ of length $2 \pi$, let $f_{a}(x)$ be the function of period $2 \pi$ and coinciding with $f(x)$ in $I_{a}$. Let

Received by the editors September 29, 1948. 


$$
\frac{1}{2} a_{0}+\sum_{n=1}^{\infty}\left(a_{n} \cos n x+b_{n} \sin n x\right)
$$

be the F.s. of $f(x)$ and

$$
s_{\omega}\left(x, f_{a}\right)=\frac{1}{2} a_{0}+\sum_{n \leqq \omega}\left(a_{n} \cos n x+b_{n} \sin n x\right), \quad \omega \geqq 0,
$$

the partial sums of (4). Then the difference

$$
\Delta_{\omega}(x)=\Delta_{\omega}(x, f, a)=S_{\omega}(x, f)-s_{\omega}\left(x, f_{a}\right)
$$

tends to zero at every point inside $I_{a}$, and the convergence is uniform in every closed interval interior to $I_{a}{ }^{1}$

Thus, tests of convergence which are valid for F.s. are immediately extensible to F.i. Moreover, since the convergence of (5) to zero implies its summability $(C, \alpha)$ for any $\alpha>0$, we immediately obtain for the summability of F.i. results analogous to those for F.s.

Now, let

$$
\int_{0}^{\infty}\{a(u) \sin u x-b(u) \cos u x\} d u
$$

be the integral conjugate to (1). The partial integrals of (6) can be written

$$
\tilde{S}_{\omega}(x, f)=-\frac{1}{\pi} \int_{-\infty}^{\infty} f(x+t) \frac{1-\cos \omega t}{t} d t
$$

if $f \in L^{p}(-\infty, \infty)$ with $1 \leqq p \leqq 2$, but again the right-hand side here has meaning only if (3) is satisfied. The following result completes (A):

(B) Let

$$
\tilde{s}_{\omega}\left(x, f_{a}\right)=\sum_{n \leqq \omega}\left(a_{n} \sin n x-b_{n} \cos n x\right), \quad \omega \geqq 1 .
$$

On the assumptions of (A), the difference

$$
\tilde{\Delta}_{\omega}(x)=\tilde{\Delta}_{\omega}(x, f, a)=\tilde{S}_{\omega}(x, f)-\xi_{\omega}\left(x, f_{a}\right)
$$

tends to a limit (not necessarily zero) at every point $x$ interior to $I_{a}$, and the convergence is uniform over any closed interval interior to $I_{a .}{ }^{2}$ T.S.

${ }^{1}$ See Zygmund, Trigonometrical series, p. 306. The book will henceforth be quoted

${ }^{2} \mathrm{Pi}$ Calleja, P., Über die Konvergenzbedingungen der komplexen Form des Fourierschen Integralen, Math. Zeit. vol. 40 (1935-1936). 
In a number of problems of the theory of F.s., we have to consider the behaviour of a F.s. differentiated term by term $k$ times. A typical result in this order of ideas is the following:

(C) Let $f_{(k)}(x)$ be the kth generalized derivative of $f(x)$. If $f_{(k)}\left(x_{0}\right)$ exists, the $F$.s. of $f(x)$, differentiated term by term $k$ times, is, at the point $x_{0}$, summable $(C, \alpha), \alpha>k$, to the value $f_{(k)}\left(x_{0}\right) .^{3}$

Similar problems exist for F.i.

In the present paper we prove a theorem that enables us to reduce a F.i. problem of the aforementioned kind to the corresponding problem on F.s., namely:

Theorem. Let $f(x)$ satisfy condition (3). Let $I_{a}=(a, a+2 \pi)$ and let $f_{a}(x)$ be the function of period $2 \pi$ coinciding with $f(x)$ in $I_{a}$. Let $S_{\omega}(x, f)$ be the partial integral (3a) and let $s_{\omega}\left(x, f_{a}\right)$ be the partial sums of the $F . s$. of $f_{a}(x)$. Let $k=1,2, \cdots$. Then the difference

$$
\frac{d^{k}}{d x^{k}} S_{\omega}(x, f)-\frac{d^{k}}{d x^{k}} s_{\omega}\left(x, f_{a}\right)
$$

is uniformly summable $(C, k)$ to zero in every closed interval interior to $I_{a}$.

Similarly, the difference

$$
\frac{d^{k}}{d x^{k}} \widetilde{S}_{\omega}(x, f)-\frac{d^{k}}{d x^{k}} \tilde{S}_{\omega}\left(x, f_{a}\right)
$$

is uniformly summable $(C, k)$ in every closed interval interior to $I_{a}$.

This theorem complements the results (A) and (B) stated above and is reduced to these results when $k=0$. An immediate application of it leads us, when (C) is taken into account, to:

$\left(\mathrm{C}^{\prime}\right)$ If $f_{(k)}\left(x_{0}\right)$ exists, the $F . i$. of $f(x)$, differentiated $k$ times under the integral sign, is, at the point $x_{0}$, summable $(C, \alpha), \alpha>k$, to the value $f_{(k)}\left(x_{0}\right)$.

In this connection we may recall the definition of summability of a series by M. Riesz's method of order $\alpha$ or summability $(R, \alpha)$ and the fact that if a series is summable to sum $s$ by the method $(R, \alpha)$, it is also summable to the same sum by the method $(C, \alpha)$ and conversely. ${ }^{4}$

We shall base the proof of the theorem on the following lemmas:

(i) Let $g(t)$ be an integrable function in $(-\pi, \pi)$ and of period $2 \pi$. Let $r>1$ and $0<\delta<\pi$. Then the integral

3 T.S. p. 257.

T.S. p. 312. 


$$
\int_{|t| \geqq \pi} \frac{g(t)}{(x-t)^{r}} d t
$$

converges absolutely and uniformly for $-\pi+\delta \leqq x \leqq \pi-\delta$.

The proof of this lemma is immediate.

(ii) Let $0<\eta<1 / 2$. Under the conditions of Lemma (i), the integral

$$
\begin{aligned}
\left\{\int_{\pi}^{\infty}+\int_{-\infty}^{-\pi}\right\} \frac{g(t)}{x-t} e^{i \omega(x-t)} d t & \\
= & \lim _{T \rightarrow \infty}\left\{\int_{\pi}^{T}+\int_{-T}^{-\pi}\right\} \frac{g(t)}{x-t} e^{i \omega(x-t)} d t
\end{aligned}
$$

converges:

(a) uniformly for $-\pi+\delta \leqq x \leqq \pi-\delta$ and any fixed $\omega$,

(b) uniformly for $-\pi+\delta \leqq x \leqq \pi-\delta, \eta \leqq \omega \leqq 1-\eta$,

(c) boundedly for $-\pi+\delta \leqq x \leqq \pi-\delta$ and any range of variation of $\omega$.

Clearly it is sufficient to suppose that $T=(2 n+1) \pi$. On such an assumption we have

$$
\begin{aligned}
\left\{\int_{\pi}^{T}+\int_{-T}^{-\pi}\right. & \} \frac{g(t)}{x-t} e^{i \omega(x-t)} d t \\
& =\sum_{k=1}^{n} \int_{-\pi}^{\pi} g(t) e^{i \omega(x-t)}\left\{\frac{e^{2 k \pi i \omega}}{2 k \pi+(x-t)}-\frac{e^{-2 k \pi i \omega}}{2 k \pi-(x-t)}\right\} d t .
\end{aligned}
$$

By direct inspection of the right-hand side of this formula we get (a) in the special case $\omega=$ an integer. Now, let $\omega$ be non-integral. Summation by parts gives

$$
\begin{aligned}
\sum_{k=1}^{\infty} \frac{e^{ \pm 2 k \pi i \omega}}{2 k \pi} & \pm(x-t) \\
= & \sum_{k=1}^{\infty}\left\{\sum_{\nu=1}^{k} e^{ \pm 2 \nu \pi i \omega}\right\}\left\{\frac{1}{2 k \pi \pm(x-t)}-\frac{1}{2(k+1) \pi \pm(x-t)}\right\} \\
= & \frac{2 \pi e^{ \pm 2 \pi i \omega}}{1-e^{ \pm 2 \pi i \omega}} \sum_{k=1}^{\infty} \frac{1-e^{ \pm 2 k \pi i \omega}}{\{2 k \pi \pm(x-t)\}\{2(k+1) \pi \pm(x-t)\}}
\end{aligned}
$$

so that

$$
\left|\sum_{k=1}^{\infty} \frac{e^{ \pm 2 k \pi i \omega}}{2 k \pi \pm(x-t)}\right|<\frac{1}{\left|1-e^{ \pm 2 \pi i \omega}\right|}\left(\frac{2}{\delta}+\frac{1}{\pi} \sum_{k=1}^{\infty} \frac{1}{k^{2}}\right),
$$

and from this result (a) and (b) follow.

To obtain (c), let us consider the expression 


$$
\begin{aligned}
& \sum_{k=1}^{n}\left\{\frac{e^{2 k \pi i \omega}}{2 k \pi+(x-t)}-\frac{e^{-2 k \pi i \omega}}{2 k \pi-(x-t)}\right\} \\
& =2 i \sum_{k=1}^{n} \frac{\sin 2 k \pi \omega}{2 k \pi-(x-t)^{2} / 2 k \pi}-2(x-t) \sum_{k=1}^{n} \frac{\cos 2 k \pi \omega}{4 k^{2} \pi^{2}-(x-t)^{2}} \\
& =2 i P_{n}(\omega, x-t)-2(x-t) Q_{n}(\omega, x-t) \text {. }
\end{aligned}
$$

Obviously $\lim _{n \rightarrow \infty} Q_{n}(\omega, x-t)$ exists uniformly for $-\pi+\delta \leqq x \leqq \pi-\delta$ and any range of variation of $\omega$. Furthermore the difference

$$
\begin{aligned}
& P_{n}(\omega, x-t)-\sum_{k=1}^{n} \frac{\sin 2 k \pi \omega}{2 k \pi} \\
& \quad=(x-t)^{2} \sum_{k=1}^{n} \frac{\sin 2 k \pi \omega}{(2 k \pi)^{2}\left\{2 k \pi-(x-t)^{2} / 2 k \pi\right\}},
\end{aligned}
$$

as $n \rightarrow \infty$, converges also uniformly for $-\pi+\delta \leqq x \leqq \pi-\delta$ and any range of variation of $\omega$. Since it is known that the series $\sum_{k=1}^{\infty} \sin 2 k \pi \omega / 2 k \pi$ is boundedly convergent in any interval of $\omega$, (c) is proved.

(iii) Let $v \neq 0$. Then for $q=1,2, \cdots ; k=1,2, \cdots$,

$$
\begin{aligned}
\frac{d^{q}}{d v^{q}} \int_{0}^{\Omega}\left(1-\frac{\omega}{\Omega}\right)^{k} e^{i \omega v} d \omega \\
=(-1)^{q} \frac{q !}{v^{q+1}} i+\sum_{\nu=0}^{k-2} \frac{(-1)^{q} k(k-1) \cdots(k-\nu)(\nu+1+q) !}{i^{\nu}(\nu+1) ! \Omega^{v+1} v^{\nu+q+2}} \\
\quad+\sum_{\nu=0}^{q-1} \frac{(-1)^{\nu} C_{q, \nu}(k+\nu) ! e^{i \Omega v}}{i^{(k+\nu+1)-q \Omega^{k+\nu-q} v^{k+\nu+1}}}+\frac{(-1)^{q}(k+q) !}{i^{k+1} \Omega^{k_{v} k+q+1}}\left(e^{i \Omega v}-1\right)
\end{aligned}
$$

where we put $\sum_{\nu=0}^{-1} \psi(\nu)=0 .^{5}$

We now need to prove that

$$
\begin{aligned}
\int_{0}^{\Omega}\left(1-\frac{\omega}{\Omega}\right)^{k} e^{i \omega v} d \omega= & -\frac{1}{i v}-\sum_{\nu=0}^{k-2} \frac{k(k-1) \cdots(k-\nu)}{\Omega^{\nu+1}(i v)^{\nu+2}} \\
& +\frac{k !}{\Omega^{k}} \frac{e^{i \Omega v}-1}{(i v)^{k+1}}
\end{aligned}
$$

because by differentiation of this formula with respect to $v$ we obtain the preceding one.

Now, (8) is certainly true for $k=1$. Let us assume that it is true for

$\checkmark(\nu)$ stands in general for any function of the non-negative integer $\nu$ appearing in this paper. 
$k=n$. We shall prove that on this hypothesis the formula holds also true for $k=n+1$. Indeed,

$$
\begin{aligned}
\int_{0}^{\Omega}( & \left.1-\frac{\omega}{\Omega}\right)^{n+1} e^{i \omega v} d \omega \\
= & {\left[\left(1-\frac{\omega}{\Omega}\right)^{n+1} \frac{e^{i \omega v}}{i v}\right]_{0}^{\Omega}+\frac{n+1}{i v \Omega} \int_{0}^{\Omega}\left(1-\frac{\omega}{\Omega}\right)^{n} e^{i \omega v} d \omega } \\
= & -\frac{1}{i v}-\sum_{\nu=0}^{n-1} \frac{(n+1) n \cdots(n+1-\nu)}{\Omega^{\nu+1}(i v)^{\nu+2}}+\frac{(n+1) !}{\Omega^{n+1}} \frac{e^{i \Omega v}-1}{(i v)^{n+2}},
\end{aligned}
$$

which was to be proved.

In particular, if $q=k$ and $A_{\nu}, B_{v}$, and $C$ are constants, (7) can be written in the form

$$
\frac{d^{k}}{d v^{k}} \int_{0}^{\Omega}\left(1-\frac{\omega}{\Omega}\right)^{k} e^{i \omega v} d \omega=(-1)^{k} \frac{k !}{v^{k+1}} i+\Phi_{\Omega}(k, v)
$$

where

$$
\begin{aligned}
\Phi_{\Omega}(k, v)= & \sum_{\nu=0}^{k-2} \frac{A_{\nu}}{\Omega^{\nu+1} v^{\nu+k+2}}+\sum_{\nu=0}^{k-1} \frac{B_{\nu}}{\Omega^{\nu} v^{\nu+k+1}} e^{i \Omega v} \\
& +\frac{C}{\Omega^{k} v^{2 k+1}}\left(e^{i \Omega v}-1\right) .
\end{aligned}
$$

These lemmas having been proved, we pass to the proof of the theorem. For the sake of simplicity, let us assume $a=0$ and let us write $f_{0}, J_{0}, J_{0}^{\prime}$ instead of $f_{a}, J_{a}, J_{a}^{\prime}$. Moreover let us put

$$
s_{\omega}^{*}(x)=s_{\omega}^{*}\left(x, f_{0}\right)=\frac{a_{0}}{2}+\sum_{n \geqq \omega}^{\prime}\left(a_{n} \cos n x+b_{n} \sin n x\right), \quad \omega \geqq 0,
$$

where the dash ' on the right indicates that if $\omega$ is an integer, we must multiply the last term by one-half. Thus, for $\omega$ positive and not integral,

$$
s_{\omega}^{*}(x)=s_{\omega}(x),
$$

and therefore instead of considering the $(C, \alpha)$ means of the difference $S_{\omega}(x)-s_{\omega}(x)$, we may consider the $(C, \alpha)$ means of the difference $S_{\omega}(x)-s_{\omega}^{*}(x)$.

Now, it is well known that if $g(t)$ is any periodic function of period $2 \pi$, integrable in the interval $(0,2 \pi)$, then ${ }^{6}$

- G. H. Hardy, Notes on some points in the integral calculus, Messenger of Mathematics vol. 52 (1922) pp. 49-53. 
$s_{\omega}^{*}(x, g)=\frac{1}{\pi} \int_{-\infty}^{\infty} g(t) \frac{\sin \omega(x-t)}{x-t} d t=\lim _{T \rightarrow \infty} \frac{1}{\pi} \int_{-T}^{T} g(t) \frac{\sin \omega(x-t)}{x-t} d t$.

Consequently, the first part of the theorem will be proved if we prove that, as $\Omega \rightarrow \infty$,

$$
\frac{k}{\pi \Omega^{k}} \int_{0}^{\Omega}(\Omega-\omega)^{k-1} d \omega \frac{d^{k}}{d x^{k}} \int_{-\infty}^{\infty}\left\{f(t)-f_{0}(t)\right\} \frac{\sin \omega(x-t)}{x-t} d t \rightarrow 0
$$

uniformly in $J_{0}^{\prime}$.

For that purpose, let $h(t)=f(t)-f_{0}(t)$, so that $h(t)=0$ in $(-\pi, \pi)$, and let us consider the integral

$$
J_{\Omega}(k, x)=\frac{k}{\pi \Omega^{k}} \int_{0}^{\Omega}(\Omega-\omega)^{k-1} d \omega \frac{d^{k}}{d x^{k}} \int_{|t| \geqq \pi} h(t) \frac{\sin \omega(x-t)}{x-t} d t .
$$

In virtue of assumption (3) and lemmas (i) and (ii), the integral

$$
\int_{|t| \geqq \pi} h(t)\left(\frac{d^{k}}{d x^{k}} \frac{e^{i \omega(x-t)}}{x-t}\right) d t
$$

is, for every fixed $\omega$, uniformly convergent in $J_{0}^{\prime}$, so that we may write, $x \in J_{0}^{\prime}$,

$$
J_{\Omega}(k, x)=\frac{k}{\pi \Omega^{k}} \int_{0}^{\Omega}(\Omega-\omega)^{k-1} d \omega \int_{|t| \geqq \pi} h(t)\left(\frac{d^{k}}{d x^{k}} \frac{\sin \omega(x-t)}{x-t}\right) d t
$$

and also

$$
J_{\Omega}(k, x)=\frac{1}{\pi} \int_{|t| \geqq \pi} h(t) d t \frac{k}{\Omega^{k}} \int_{0}^{\Omega}(\Omega-\omega)^{k-1}\left(\frac{d^{k}}{d x^{k}} \frac{\sin \omega(x-t)}{x-t}\right) d \omega,
$$

the inversion of integrations being justified by (3) and lemmas (i) and (ii).

Integrating the inner integral by parts, we get

$$
\begin{aligned}
J_{\Omega}(k, x) & =\frac{1}{\pi} \int_{|t| \geqq \pi} h(t) d t \int_{0}^{\Omega}\left(1-\frac{\omega}{\Omega}\right)^{k}\left(\frac{d^{k}}{d x^{k}} \cos \omega(x-t)\right) d \omega \\
& =\frac{1}{\pi} \int_{|t| \geqq \pi} h(t) d t \frac{d^{k}}{d x^{k}} \int_{0}^{\Omega}\left(1-\frac{\omega}{\Omega}\right)^{k} \cos \omega(x-t) d \omega \\
& =\frac{1}{\pi} \Re\left\{\int_{|t| \geqq \pi} h(t) d t \frac{d^{k}}{d x^{k}} \int_{0}^{\Omega}\left(1-\frac{\omega}{\Omega}\right)^{k} e^{i \omega(x-t)} d \omega\right\}
\end{aligned}
$$

or, according to (9), 


$$
J_{\Omega}(k, x)=\frac{1}{\pi} \Re\left\{\int_{|t| \geqq \pi} h(t) \Phi_{\Omega}(k, x-t) d t\right\} .
$$

Let us consider the integral $\int_{\left.|t|\right|_{\pi}} h(t) \Phi_{\Omega}(k, x-t) d t$ and let us suppose we have replaced $\Phi_{\Omega}(k, x-t)$ by its expression (10). If so, we shall obtain integrals belonging to any one of the following types:

$$
\begin{gathered}
\frac{1}{\Omega^{s}} \int_{|t| \geqq \pi} \frac{h(t)}{(x-t)^{r}} d t ; \quad \frac{1}{\Omega^{s}} \int_{|t| \geqq \pi} \frac{h(t)}{(x-t)^{r}} e^{i \Omega(x-t)} d t ; \\
\int_{|t| \geqq \pi} \frac{h(t)}{(x-t)^{2}} e^{i \Omega(x-t)} d t
\end{gathered}
$$

where $s$ and $r$ are positive integers such that $s \geqq 1, r \geqq 3$.

Clearly every integral belonging to either of the two first types tends to zero uniformly in $J_{0}^{\prime}$ as $\Omega \rightarrow \infty$. As for $\int_{|t| \geqq x}\left(h(t) /(x-t)^{2}\right) e^{d \Omega(x-t)} d t$, we have

$$
\begin{aligned}
\int_{|t| \geqq \pi} \frac{h(t)}{(x-t)^{2}} e^{i \Omega(x-t)} d t & =\left\{\int_{A \geqq|t| \geqq \pi}+\int_{|t| \geqq A}\right\} \frac{h(t)}{(x-t)^{2}} e^{i \Omega(x-t)} d t \\
& =I_{1}+I_{2} .
\end{aligned}
$$

If we choose $A$ large enough, by (3) and Lemma (i), $\left|I_{2}\right|<\epsilon / 2$ for any $x \in J_{0}^{\prime}$. On the other hand, $1 /(x-t)^{2}$ being for $x \in J_{0}^{\prime}, \pi \leqq|t| \leqq A$ a uniformly bounded and uniformly continuous function depending on the parameter $x, I_{1} \rightarrow 0$ as $\Omega \rightarrow \infty$ uniformly in $J_{0}^{\prime}$, so that

$$
\int_{|t| \geqq \pi} \frac{h(t)}{(x-t)^{2}} e^{i \Omega(x-t)} d t \rightarrow 0
$$

uniformly in $J_{0}^{\prime}$ and the first part of the theorem is proved. For the second part, we begin by putting

$$
\tilde{s}_{\omega}^{*}(x)=\tilde{s}_{\omega}^{*}\left(x, f_{0}\right)=\sum_{n \leqq \omega}^{\prime}\left(a_{n} \sin n x-b_{n} \cos n x\right), \quad \omega \geqq 1,
$$

where the dash ' on the right has the same meaning as before. Now, by analogy with $s_{\omega}^{*}(x)$, the expression $s_{\omega}^{*}(x)$ can be written in the form

$$
\begin{aligned}
\xi_{\omega}^{*}(x) & =-\frac{1}{\pi} \int_{-\infty}^{\infty} f_{0}(t) \frac{1-\cos \omega(x-t)}{x-t} d t \\
& =-\lim \frac{1}{\pi} \int_{-T}^{T} f_{0}(t) \frac{1-\cos \omega(x-t)}{x-t} d t,
\end{aligned}
$$


and the device used in the first part of the theorem entitles us to write

$$
\begin{aligned}
\frac{k}{\pi \Omega^{k}} \int_{0}^{\Omega}(\Omega & -\omega)^{k-1} \frac{d^{k}}{d x^{k}}\left(\tilde{S}_{\omega}(x)-\tilde{s}_{\omega}(x)\right) d \omega \\
= & -\frac{1}{\pi} \Im\left\{\int_{|t| \geqq \pi} h(t) d t \frac{d^{k}}{d x^{k}} \int_{0}^{\Omega}\left(1-\frac{\omega}{\Omega}\right)^{k} e^{i \omega(x-t)} d \omega\right\} \\
= & \frac{(-1)^{k+1} k !}{\pi} \int_{|t| \geqq \pi} \frac{h(t)}{(x-t)^{k+1}} d t \\
& -\frac{1}{\pi} \Im\left\{\int_{|t| \geqq \pi}(t) \Phi_{\Omega}(k, x-t) d t\right\},
\end{aligned}
$$

and inasmuch as $\int_{|t| \geqq x} h(t) \Phi_{\Omega}(k, x-t) d t \rightarrow 0$ uniformly in $J_{0}^{\prime}$, we obtain the result

$$
\begin{aligned}
\lim _{\Omega \rightarrow \infty} \frac{k}{\pi \Omega^{k}} \int_{0}^{\Omega}(\Omega-\omega)^{k-1} \frac{d^{k}}{d x^{k}}\left(\tilde{S}_{\omega}(x)\right. & \left.-\tilde{s}_{\omega}(x)\right) d \omega \\
& =\frac{(-1)^{k+1} k !}{\pi} \int_{|t| \geqq \pi} \frac{h(t)}{(x-t)^{k+1}} d t
\end{aligned}
$$

uniformly in $J_{0}^{\prime}$, and this completes the proof of the theorem. Before ending, the author wishes to express to Professor A. Zygmund his gratitude for the assistance and encouragement shown to him while he was engaged on the present paper during his tenure of a scholarship at the University of Pennsylvania in 1946-1947.

UNIVERSITY OF TUCUMÁN AND

University of Pennsylvania 